\title{
Instability of crack propagation in brittle bulk metallic glass
}

\author{
G. Wang ${ }^{\text {a }}$, K.C. Chan ${ }^{\text {a,* }}$, X.H. Xu ${ }^{\text {b }}$, W.H. Wang ${ }^{\mathrm{c}}$ \\ ${ }^{a}$ Department of Industrial and Systems Engineering, The Hong Kong Polytechnic University, Yuk Choi Road, Hung Hom, Kowloon, Hong Kong, China \\ ${ }^{\mathrm{b}}$ State Key Laboratory of Non-linear Mechanics, Institute of Mechanics, Chinese Academy of Sciences, Beijing 100080, China \\ ${ }^{\mathrm{c}}$ Institute of Physics, Chinese Academy of Sciences, Beijing 100080, China
}

Received 2 May 2008; accepted 3 August 2008

Available online 31 August 2008

\begin{abstract}
The instability of the crack tip in brittle Mg-based bulk metallic glass (BMG) is studied. The formation of various fractographic surfaces of the BMG is associated with the instability of the fluid meniscus, which is due to viscous fluid matter being present on the fracture process zone. Depending on the values of the wavelength of the initial perturbation of the fluid meniscus and the local stress intensity factor, different fracture surface profiles, i.e. a dimple-like structure, a periodic corrugation pattern and a pure mirror zone are formed. The fractogaphic evolution is significantly affected by the applied stress. A decreased fracture surface roughness is observed under a low applied stress. An increased fracture surface roughness, which has frequently been reported by other researchers, is also observed in the present studies under a high applied stress. Unique fractographic features are attributed to the non-linear hyperelastic stiffening (or less softening) mechanism.
\end{abstract}

(C) 2008 Acta Materialia Inc. Published by Elsevier Ltd. All rights reserved.

Keywords: Metallic glasses; Fracture; Scanning electron microscopy; Atomic force microscopy

\section{Introduction}

A fracture in brittle glass is traditionally referred to as a two-dimensional (2D) surface formed by a crack moving along a planar, straight-line path. In reality, however, the fracture has a complicated morphology, and the moving crack results in a three-dimensional (3D) acoustic wave, crack oscillating and branching, which do not exist in the classical linear theories of crack propagation [1-4]. The fracture surface roughening phenomena of brittle glassy materials have generated much interest recently, and intensive work has been carried out to uncover the mechanisms governing the dynamics of crack and the connection between fractographic structure and fracture energy dissipation [5,6]. Driven by the elastic energy stored in a stressed elastic body, brittle cracks exhibit an increased roughening of the fracture surface owing to the dynamic

\footnotetext{
* Corresponding author. Tel.: +852 27664981; fax: +85223625267

E-mail address: mfkcchan@polyu.edu.hk (K.C. Chan).
}

instability of the crack front [7]. Cracks moving at low speeds create flat mirror surfaces. At higher speeds, dynamic cracking generally brings out instability in the crack tip, sprouting small side cracks, termed microbranches [8]. This then results in a rougher surface (mist zone) and finally leads to a very rough, irregularly faceted (hackle) surface [7,9]. Attempts to relate this energy flux to the response marks (roughness) on the crack surface have been extensively carried out in the framework of linear elasticity theory [10-16]. However, in real materials, such as in metals, the crack tip always exhibits non-linear behavior $[17,18]$, and the precise physical mechanism for the formation of fracture surface roughness and the origin of crack dynamic instability remain unclear.

Previous fracture studies of brittle materials have focused on brittle glassy polymers, oxide glasses and polymer gels [1-4]. Recently, several families of brittle bulk metallic glasses (BMG), such as Mg-based [19,20], Fe-based [21,22] and rare-earth-based BMG [23], have been developed. Compared with previous tough BMG with notch fracture toughness $\left(K_{\mathrm{c}}\right)$ value $>20 \mathrm{MPa} \mathrm{m}^{1 / 2}[24]$, 
brittle BMG have extremely low $K_{\mathrm{C}}$ values of $<2 \mathrm{MPa} \mathrm{m}{ }^{1 / 2}$, which are very close to those of oxide glasses [24]. The unique atomic-scale disordered structure in BMG generates a dimple-like structure [25] and a periodic corrugation pattern [26-29] presenting on the fracture surface. The formations of the dimple-like structure and the periodic corrugation pattern suggest that the plastic deformation in the crack tip of the brittle BMG dominates the cracking process $[25,26]$. Recently, a competition process between the local plastic softening mechanism and the local cleavage mechanism has been proposed to elucidate the energy dissipation in BMG fracturing [30]. As the fracture behavior is a dynamic process, its fractographies will evolve as the crack propagates due to the changes in loading strain-rate [30], crack propagation speed [22] and the dynamic effects [27]. These factors will seriously affect the energy dissipation mechanism. So far, the evolution of fractured surfaces with crack propagation has, however, been less studied, although this phenomenon is highly important in understanding the fracture energy dissipation and the micromechanism of brittle cracking $[7,16,30]$. In particular, the way in which the stress field influences the energy dissipation in the crack tip has not been reported. In this paper, the fractographic evolution and the corresponding dissipation mechanisms of the fracture energy during crack propagation in a $\mathrm{Mg}_{65} \mathrm{Cu}_{25} \mathrm{Gd}_{10}$ BMG are quantitatively analyzed.

\section{Experimental procedures}

Alloy ingots were prepared by induction melting a mixture of pure metal elements, followed by argon gas injec- tion casting into $\mathrm{Cu}$ molds to form rod-like BMG specimens $5 \times 3 \times 60 \mathrm{~mm}$ and $\varnothing 3 \times 60 \mathrm{~mm}$ in size. The glassy nature of the $\mathrm{Mg}_{65} \mathrm{Cu}_{25} \mathrm{Gd}_{10}$ BMG was checked by differential scanning calorimetry (DSC) and X-ray diffraction (XRD). The tension and three-point bending tests of the $\mathrm{Mg}_{65} \mathrm{Cu}_{25} \mathrm{Gd}_{10}$ BMG were carried out in an Instron 5565-type machine with a crosshead moving speed of $1 \mathrm{~mm} \mathrm{~min}{ }^{-1}$. To avoid exceptional cases being taken as typical, both tension and bending tests were repeated four times. A dog-bone-shaped specimen was machined to perform the tension test. The gauge size of the tension specimens was $\varnothing 1.6 \times 4 \mathrm{~mm}$. The nominal tension stressstrain curve is shown in Fig. 1a. The specimens for the pre-notched three-point bending tests had a geometric size $16 \times 3 \times 5 \mathrm{~mm}$. A single edge notch with diameter $225 \mu \mathrm{m}$ and depth $\sim 550-650 \mu \mathrm{m}$ was induced by a diamond saw. The load-displacement curve for the pre-notched bending test is shown in Fig. 1b (as the extensometer was not used in the bending test, the stress-strain curve cannot be plotted). The geometric size of the specimens for the threepoint bending test, without notches, is the same as that of the pre-notched specimens. The load-displacement curve for the bending test is shown in Fig. 1c. The average fracture stresses $\left(\sigma_{\mathrm{F}}\right)$ for the pre-notched three-point bending, three-point bending and tension are $122 \mathrm{MPa}$, $702 \mathrm{MPa}$ and $732 \mathrm{MPa}$, respectively. The elastic modulus $(E)$ is $44.7 \mathrm{GPa}$. The newly generated crack surface was observed by scanning electron microscopy (SEM) with a JEOL JSM-6335F instrument and a Digital Instrument NanoScope IV atomic force microscope operated at a tapping mode. To remove the influence of residual stress on the atomic force microscopy (AFM) image, the fractured
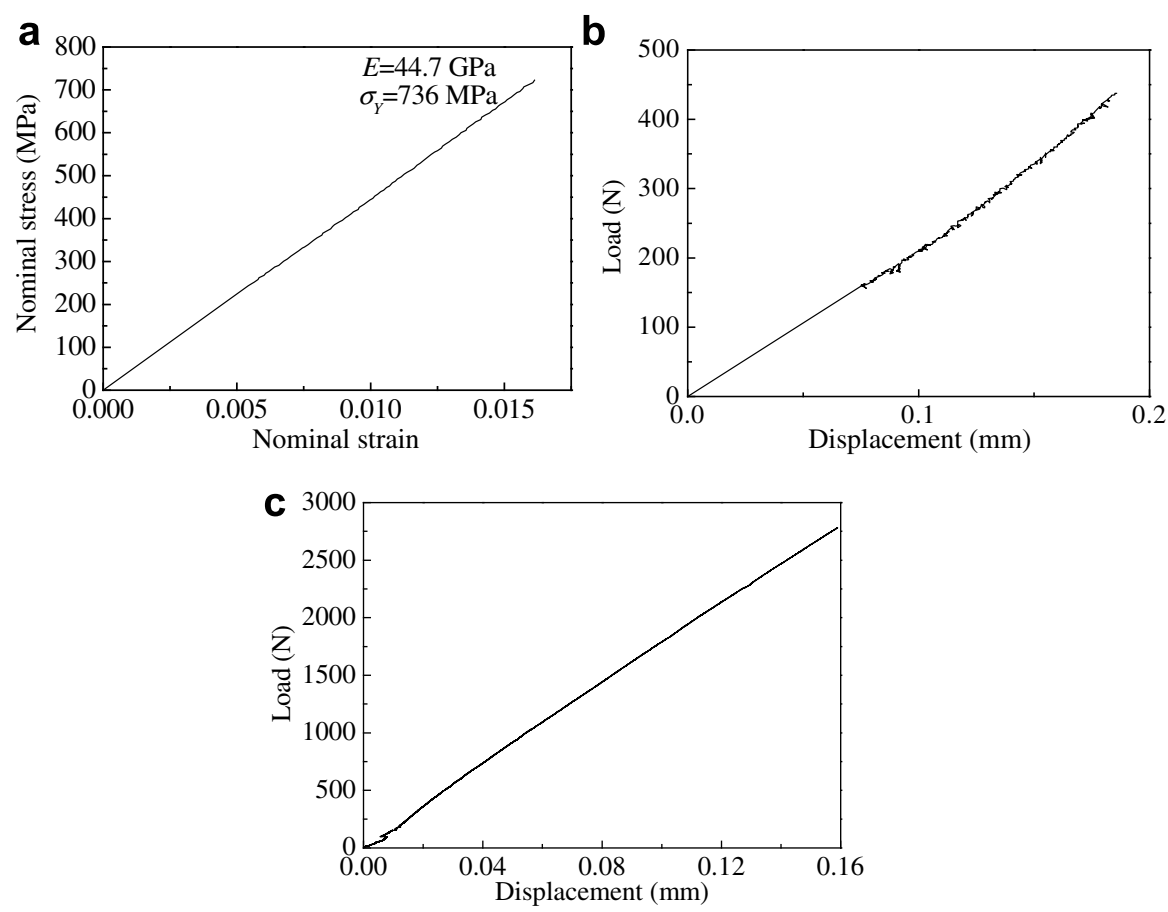

Fig. 1. (a) Tension nominal stress-strain curve; (b) pre-notched three-point bending load-displacement curve; (c) three-point bending load-displacement curve. 
BMG was sealed in a quartz tube under vacuum (gas pressure $10^{-2} \mathrm{~Pa}$ ) and then annealed at $353 \mathrm{~K}$ for $10 \mathrm{~min}$.

\section{Results}

\subsection{SEM observations}

The fracture surface profile for the pre-notched threepoint bent BMG is shown in Fig. 2. Four different zones can be discerned along the crack propagation direction. The fractographic evolution sequence is a dimple-like structure zone (Fig. 2c), a dimple and corrugation mixture zone (Fig. 2c and d), a periodic corrugation pattern zone
(Fig. 2e and f) and a featureless mirror zone (Fig. 2g). It is evident that the dimple-like structure is eventually replaced by the periodic corrugation pattern during the crack front sweeping the mixture zone (Fig. 2c and d). In the corrugation pattern zone, the spacing of the corrugation decreases to almost zero with the crack propagation in the corrugation zone, and then the featureless mirror zone appears (Fig. 2e-g). A fractographic evolution profile is drawn in Fig. $2 \mathrm{~h}$.

The fractography of the tension fractured BMG is different from that of the bending fractured BMG, as shown in Fig. 3. The fractography shows an evolving sequence of the periodic corrugation pattern (Fig. $3 b$ and c), the mixture
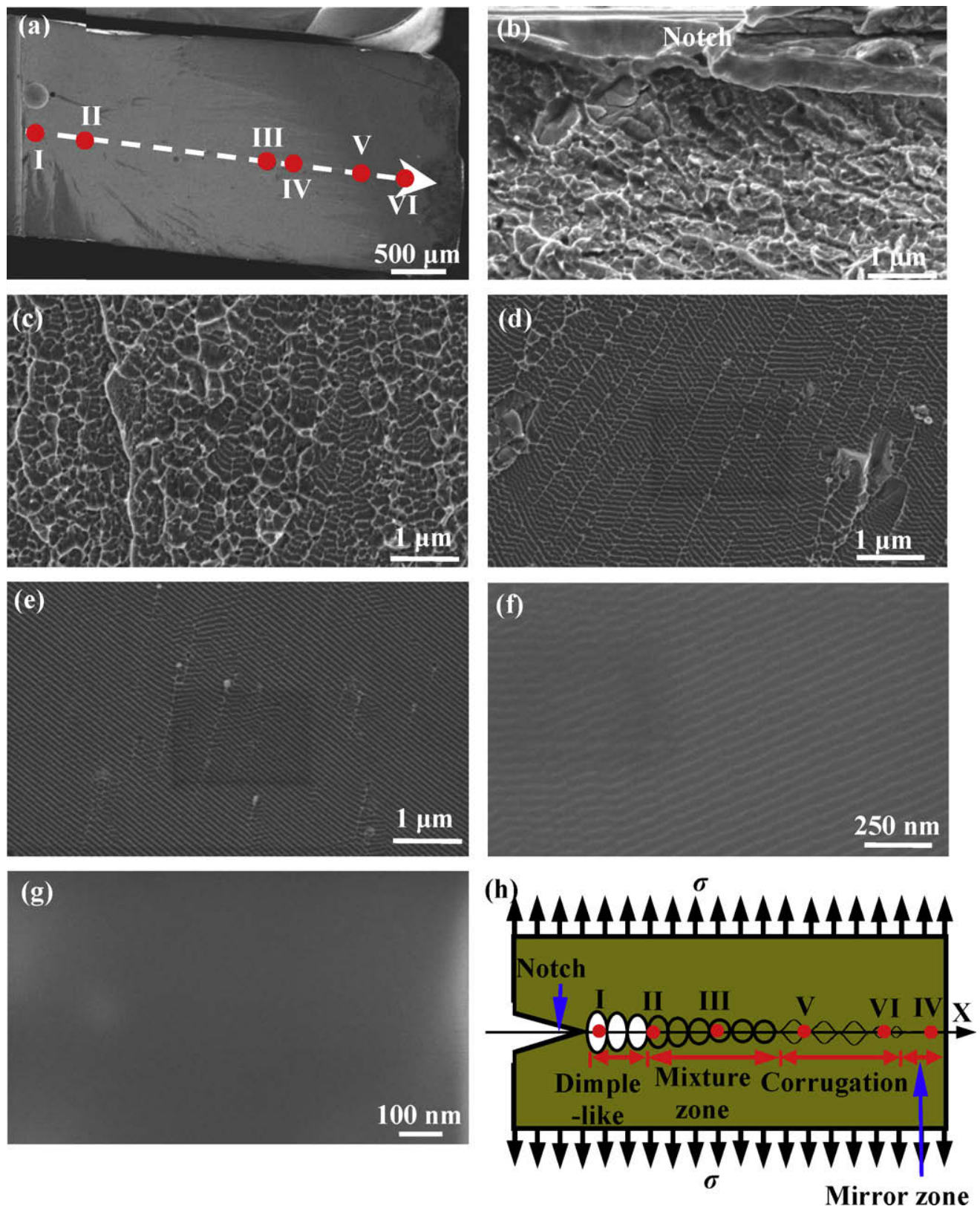

Fig. 2. Fractographic evolution for the Mg-based BMG under pre-notched three-point bending test: (a) overview (arrow indicates crack propagation direction); (b) dimple-like structure on Point I in (a) (notch region); (c) dimple and corrugation mixture zone at Point II in (a); (d) dimple and corrugation mixture zone at Point III in (a); (e) periodic corrugation pattern at Point IV in (a); (f) periodic corrugation pattern at Point V in (a); (g) pure mirror zone at Point VI in (a); (h) sketch of fractographic evolution on the fracture surface. 
zone (Fig. 3d), the dimple-like structure (Fig. 3e) and the hackle zone (Fig. 3f and g). This fractographic evolution can be classified as taking place in three zones, i.e. a mirror zone (the corrugation pattern zone), a mist zone (the dimple-like structure) and a hackle zone. Along the crack propagation, the spacing of the periodic corrugation pattern increases from $\sim 34 \mathrm{~nm}$ to $80 \mathrm{~nm}$ in the mirror zone, and the size of the dimple-like structure increases from $\sim 80 \mathrm{~nm}$ to $500 \mathrm{~nm}$ in the mist zone. The evolution trend of the fractography is contrary to that in the pre-notched bending case. The hackle zone formation generates a multiple layered fractured surface (Fig. 3f). Looking more closely at the details of the hackle zone, it can be observed that it is composed of a dimple-like structure and has a periodic corrugation pattern. A transformation from the dimple-like structure and the periodic corrugation is observed in the center of the hackle zone (Fig. 3h), where the dimple-like structure is reduced in size. As the dimple size decreases to $\sim 70 \mathrm{~nm}$, the dimple-like structure will be replaced by the periodic corrugation pattern and will spread to the edge of the hackle surface with the spacing reducing from $\sim 70$ to $37 \mathrm{~nm}$. A fractographic evolution profiled is drawn in Fig. 3i.

The fractographic evolution along the crack propagation of the three-point bending fractured BMG without the notch is almost same as that of the tension fractured BMG (Fig. 4). The evolution sequence is also the mirror zone (the periodic corrugation pattern) (Fig. $4 \mathrm{~b}$ and c), the mist zone (the dimple-like structure) (Fig. 4e) and the hackle zone (Fig. $4 \mathrm{f}$ and g). The evolution trend of the fractography in the bending fractured BMG without the notch is also the same as that which occurs in the tension fractured $\mathrm{BMG}$, i.e. the spacing of the corrugation pattern and the size of the dimple-like structure increase as the crack propagates. The features of the dimple-like structure evolution in the hackle zone are also the same as that in the tension case. The profile of the fractographic evolution is sketched in Fig. 4h.

\subsection{AFM observations}

Further quantitative observations on the fracture surface roughness for the pre-notched bending fractured $\mathrm{BMG}$ and the tension fractured $\mathrm{BMG}$ are carried out by AFM, which are shown in Figs. 4 and 5, respectively. As the fractographic evolution of the bending fractured BMG without the notch is the same as that which happens in the tension fractured BMG, the AFM images for the bending fractured BMG without the notch are not shown.

In the pre-notched bending fractured BMG, the AFM observation points are along the crack propagation direction, i.e. along the dash arrow in Fig. 2a, with an interval distance of $\sim 500 \mu \mathrm{m}$. Four AFM images captured in the four different zones are chosen to reflect the fractographic evolution profile, as shown in Fig. 5, in which the fracture surface is on the $X-Y$ plane. In the dimple-like structure zone, the dimple size and the dimple depth are inhomoge- neous (Fig. 5a). They range from $620 \mathrm{~nm}$ to $100 \mathrm{~nm}$ and from $200 \mathrm{~nm}$ to $10 \mathrm{~nm}$, respectively (Fig. 5b). The average values of the dimple size and depth are $545 \mathrm{~nm}$ and $88 \mathrm{~nm}$, respectively. In the mixture zone, both the dimple-like structure with large roughness and a relatively flat corrugation pattern are observed (Fig. 5c). The average dimple size decreases from $570 \mathrm{~nm}$ to $150 \mathrm{~nm}$, and the depth reduces from $80 \mathrm{~nm}$ to $20 \mathrm{~nm}$ as the crack propagates in the mixture zone (Fig. $5 \mathrm{~d}$ and Table 1). Then the dimple-like structure is replaced by the corrugation pattern. In the periodic corrugation zone, the sectional shape on the $X-Z$ plane, i.e. along the crack propagation direction, exhibits a sinusoidlike shape with spacing $34 \mathrm{~nm}$ as shown in section A-A in Fig. 5f. The sectional shapes on the $Y-Z$ plane, i.e. perpendicular to the crack propagation direction and along the peak and the valley of the corrugation, also exhibit a wavy shape with wavelength $37 \mathrm{~nm}$ (section B-B and section C$\mathrm{C}$ in Fig. 5f). Furthermore, the sectional shape and the wavelength along the valley of the corrugation are consistent with those along the peak of the corrugation (section $\mathrm{B}-\mathrm{B}$ and section $\mathrm{C}-\mathrm{C}$ in Fig. 5f). The spacing of the corrugation pattern decreases from $\sim 100 \mathrm{~nm}$ to $30 \mathrm{~nm}$, and the depth decreases from $7 \mathrm{~nm}$ to $4.2 \mathrm{~nm}$ as the crack propagates (Table 1). In the mirror zone, the roughness of the surface is $<1 \mathrm{~nm}$, and no dimple-like structure or periodic corrugation pattern can be found (Fig. $5 \mathrm{~g}$ and h). The dimple size and corrugation spacing, depths of the dimple structure and the periodic corrugation measured from the AFM images are listed in Table 1.

As for the tension fractured BMG, a series of AFM measurements are also carried out following the dashed arrow in Fig. 3a with an interval distance of $\sim 100 \mu \mathrm{m}$. All the results measured from the AFM images are also listed in Table 1. Four representative AFM images are shown in Fig. 6. These results are consistent with those observed by SEM (cf. Fig. 3), i.e. the sequence of the fractographic evolution is the periodic corrugation pattern zone (Fig. 6a and c), the mixture zone (Fig. 6e) and the dimple-like structure (Fig. 6g). However, owing to the large undulation in the hackle zone, the surface morphology in this zone cannot be measured by AFM. It is evident that the fracture surface exhibits an increased surface roughness, characterized by the depth of the dimple-like structure or the corrugation pattern increasing as the crack propagates. When the crack propagates from the crack nucleation spot to the region near the hackle zone, the depth of the dimple-like structure and the corrugation pattern increases from $\sim 3.2 \mathrm{~nm}$ to $84 \mathrm{~nm}$ (Table 1), which is totally different from what is happening in the bending fractured BMG.

\section{Analysis}

\subsection{Crack propagation process}

The nature of the metallic bond structure in the glassy phase suggests that BMG exhibit primarily metallic charac- 

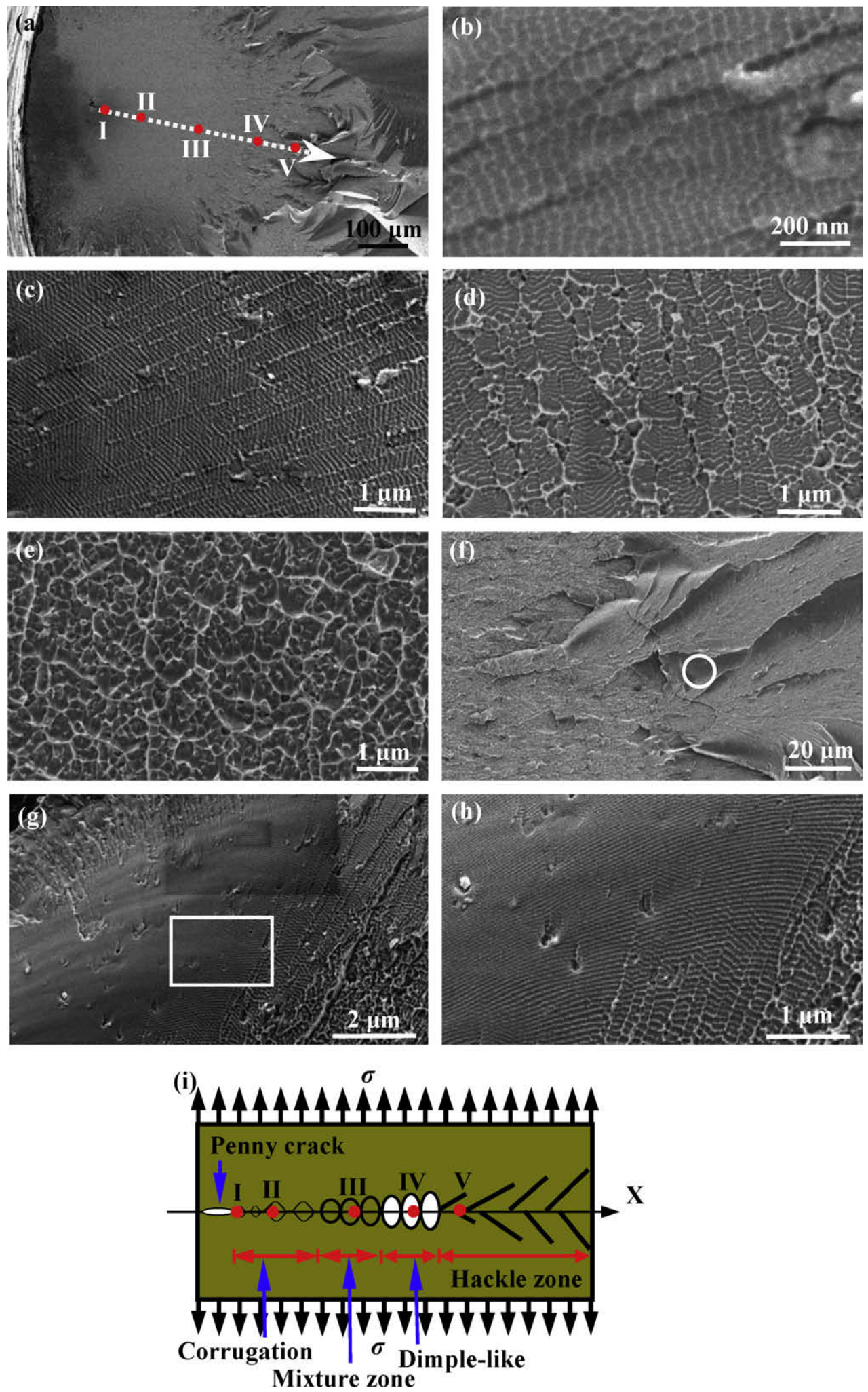

Fig. 3. Fractographic evolution for the Mg-based BMG under the tension test: (a) overview (arrow indicates crack propagation direction); (b) corrugation pattern at Point I in (a), i.e. the crack nucleation spot; (c) periodic corrugation pattern at Point II in (a); (d) dimple and corrugation mixture zone at Point III in (a); (e) dimple zone at Point IV in (a); (f) hackle zone at Point V in (a); (g) surface of hackle zone corresponding to the area circled in (e); (h) corrugation pattern on the surface of the hackle zone, corresponding to the area squared in (g); (i) sketch of fractographic evolution on the fracture surface.

ter, i.e. strain can be accommodated at the atomic-scale [30]. The metallic bond reformation driven by strain would result in a plastic flow or softening at the nanoscale [31]. Previous studies have confirmed that the dimple-like 

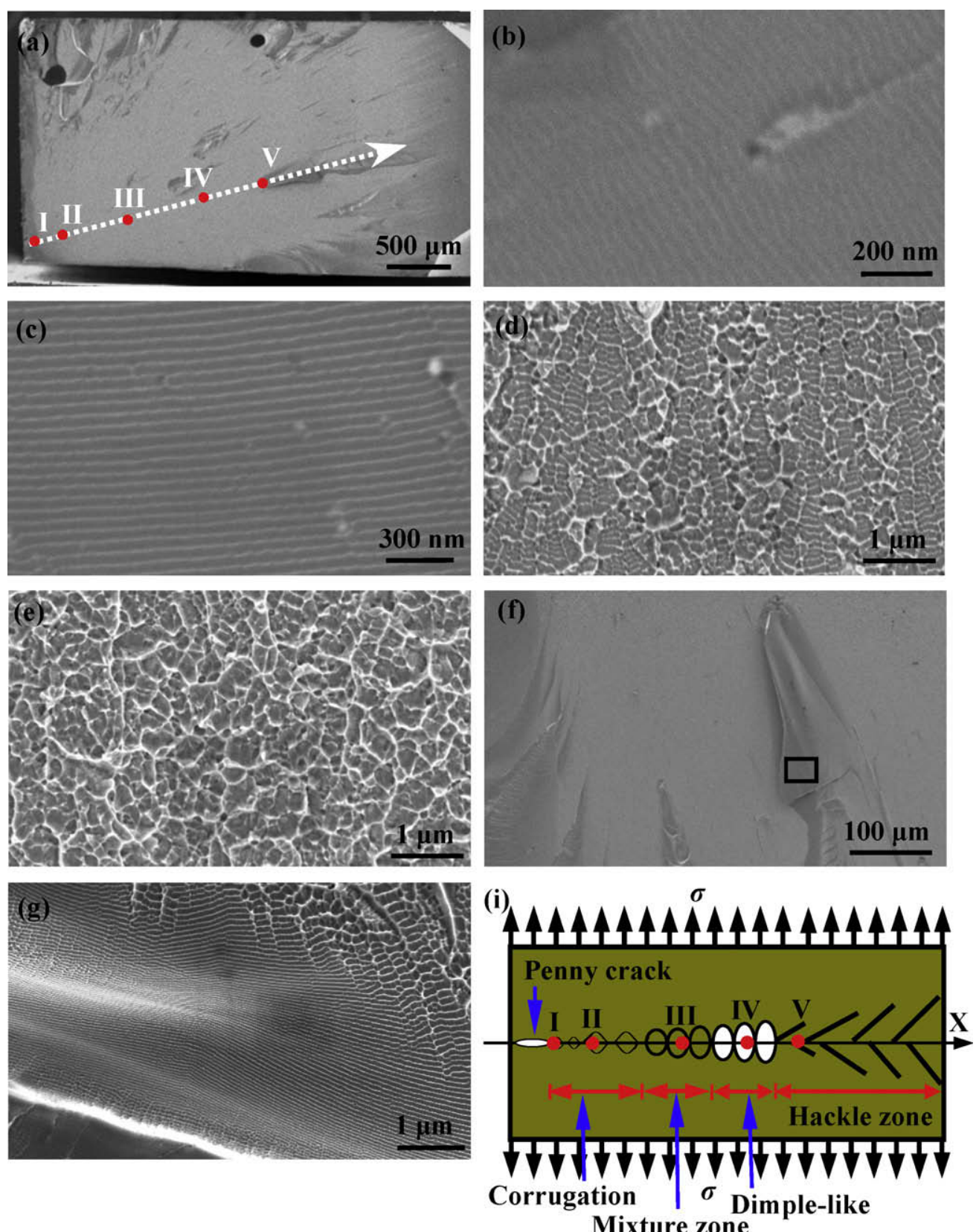

Fig. 4. Fractographic evolution for the Mg-based BMG under the three-point bending test: (a) overview (arrow indicates crack propagation direction); (b) periodic corrugation pattern at Point I in (a), i.e. the crack nucleation spot; (c) periodic corrugation pattern at Point II in (a); (d) dimple and corrugation mixture zone at Point III in (a); (e) dimple zone at Point IV in (a); (f) hackle zone at Point V in (a); surface of hackle zone corresponding to the area circled in (e); (g) corrugation pattern on the surface of the hackle zone, corresponding to the area squared in (f); (h) sketch of fractographic evolution on the fracture surface.

structure and the periodic corrugation pattern are due to the local plastic flow (softening) occurring ahead of the crack tip during their formation processes. These processes are characterized by the peak to peak matching on two opposite fracture surfaces $[25,26]$. Therefore, the local plastic flow plays a dominant role in blunting initially sharp cracks where progressive local separation still occurs by the coalescence of the damage cavities along the extension of the crack plane. The crack propagation process is shown in Fig. 7.
According to the Irwin-Orowan small-scale zone model [32], a crack blunts in a semicircle-like shape as shown in Fig. $7 \mathrm{a}$, and the fracture surface separation processes are confined to a small zone in the front of the crack tip, defined as the fracture process zone with radius $R$. The crack tip open displacement (CTOD) at the intersection of a $90^{\circ}$ vertex with the crack flanks is also plotted in Fig. 7a, which is associated with the critical stress intensity factor. As the glassy phase in the fracture process zone can be treated as a viscous fluid owing to local temperature 
a

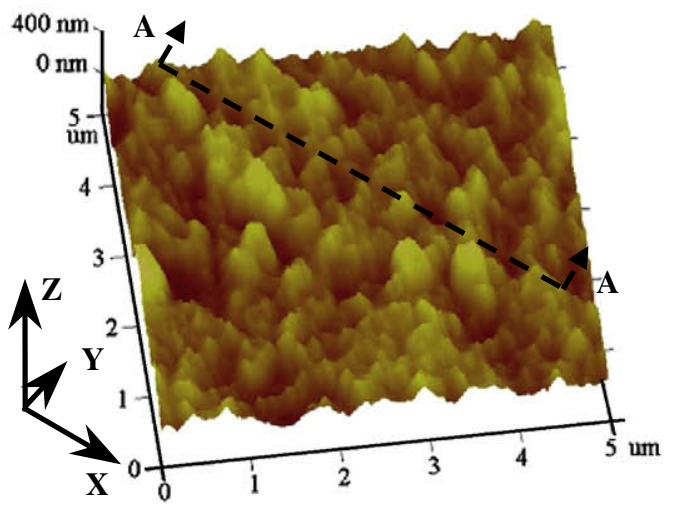

b
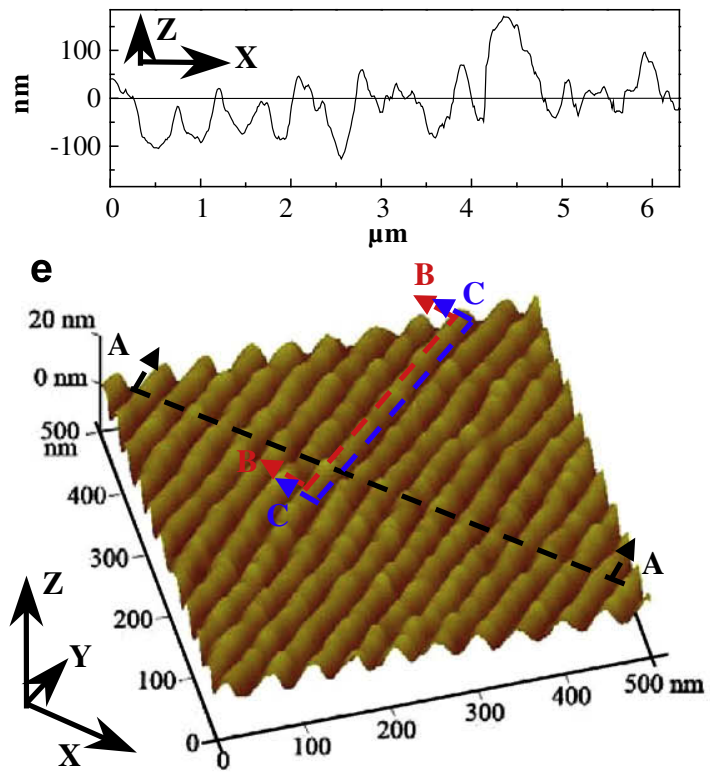

$$
\text { f }
$$

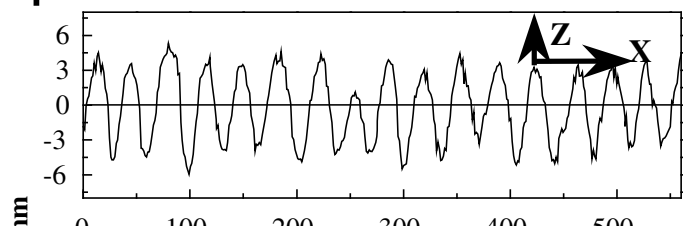

$\Xi$

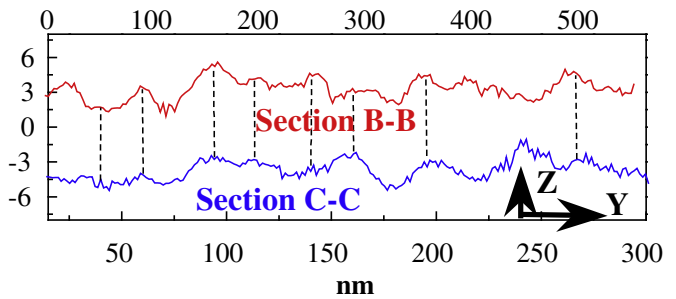

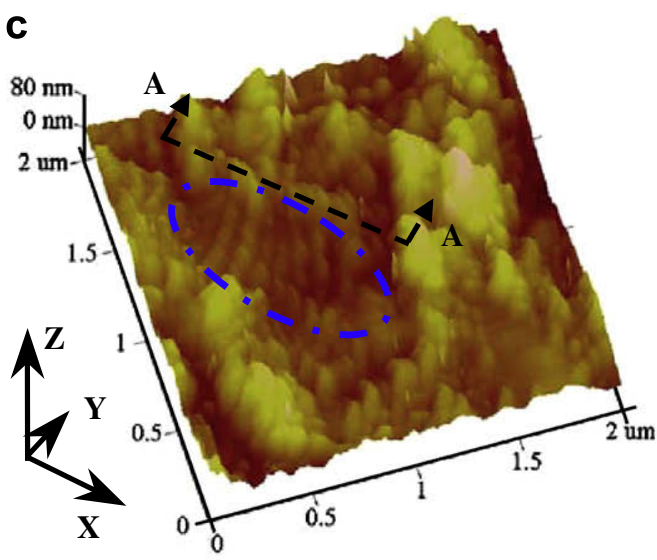
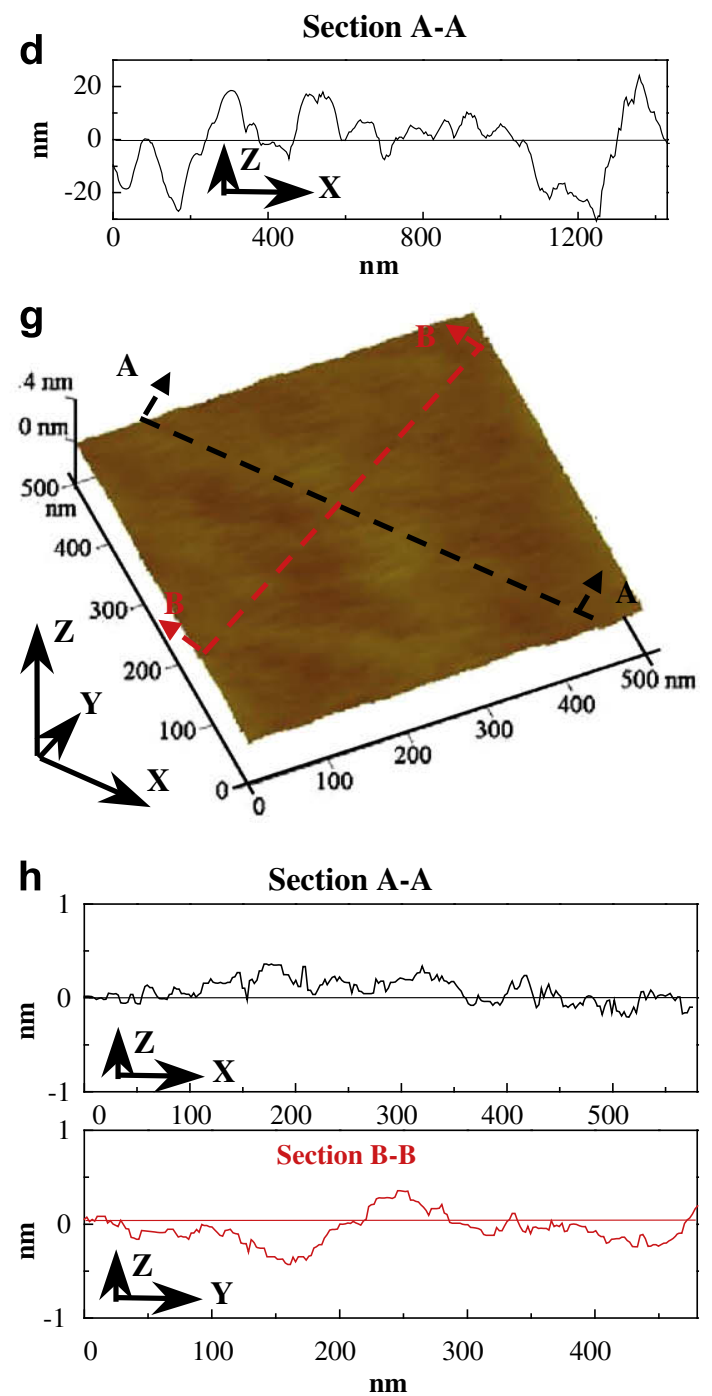

Fig. 5. AFM images for the fractography of the pre-notched three-point bending fractured BMG. Crack propagation direction is along the $X$-axis. (a) $3 \mathrm{D}$ morphology for the dimple-like structure. (b) Section A-A is the sectional shape of the dimple-like structure in (a). (c) 3D morphology for the mixture zone. The periodic corrugation pattern is circled by a dash-dot line. (d) Section A-A is the sectional shape of the dimple-like structure in (c). (e) 3D morphology for the periodic corrugation pattern. (f) Section A-A is the sectional shape of the corrugation in (e). Section B-B and Section C-C are the sectional shapes of the peak and the valley of the corrugation in (e), respectively. (g) 3D morphology for the mirror zone. (h) Section A-A and section B-B are the sectional shapes perpendicular to each other in the mirror zone in $(\mathrm{g})$.

elevation accompanying plastic deformation near the crack tip [33], a fluid meniscus will be shaped in the crack tip. Local stress generates a negative pressure $\left(\sigma_{\mathrm{N}}\right)$ in the frac- ture process zone [34]. The balance between the surface tension of the viscous fluid and the negative pressure generates a curved fluid surface, called a fluid meniscus. The negative 
Table 1

Data measured from the AFM results for the fracture surfaces

\begin{tabular}{|c|c|c|c|c|c|c|c|}
\hline$L(\mu \mathrm{m})$ & $d_{\mathrm{S}}(\mathrm{nm})$ & $d_{\mathrm{D}}(\mathrm{nm})$ & $K_{\mathrm{C}}\left(\mathrm{MPa} . \mathrm{m}^{0.5}\right)$ & $\lambda_{\mathrm{F}}(\mathrm{nm})$ & $R(\mathrm{~nm})$ & $\lambda_{\mathrm{I}}(\mathrm{nm})$ & Surface marks \\
\hline \multicolumn{8}{|c|}{ Three-point bending fractured $B M G$} \\
\hline$<100^{\mathrm{a}}$ & 545 & 88 & 2.595 & 545 & 660 & 263 & Dimple-like \\
\hline$\sim 400$ & 520 & 78 & 2.443 & 520 & 585 & 247 & Mixture zone \\
\hline$\sim 900$ & 211 & 34 & 1.613 & 211 & 255 & 163 & Mixture zone \\
\hline$\sim 1400^{\mathrm{b}}$ & 156 & 21 & 1.268 & 156 & 157 & 128 & Mixture zone \\
\hline$\sim 2100$ & 142 & 19 & 1.206 & 142 & 142 & 122 & Mixture zone \\
\hline$\sim 2500$ & 56 & 6.9 & 0.727 & 52 & 52 & 74 & Corrugation \\
\hline$\sim 2800$ & 36 & 4.7 & 0.600 & 37 & 35 & 60 & Corrugation \\
\hline$\sim 3000^{\mathrm{c}}$ & 34 & 4.4 & 0.580 & 37 & 32 & 58 & Corrugation \\
\hline$\sim 3600$ & 32 & 4.2 & 0.567 & 33 & 31 & 57 & Corrugation \\
\hline$\sim 4000^{\mathrm{d}}$ & - & - & - & - & - & & Mirror \\
\hline \multicolumn{8}{|c|}{ Tension fractured $B M G$} \\
\hline$\sim 0^{\mathrm{e}}$ & 31 & 3.9 & 0.545 & 35 & 29 & 55 & Dimple-like \\
\hline$\sim 100^{\mathrm{f}}$ & 36 & 5.0 & 0.619 & 44 & 37 & 62 & Corrugation \\
\hline$\sim 200^{\mathrm{g}}$ & 118 & 25 & 1.383 & 118 & 187 & 140 & Mixture zone \\
\hline$\sim 300$ & 361 & 49 & 1.936 & 361 & 367 & 196 & Dimple-like \\
\hline$\sim 400^{\mathrm{h}}$ & 554 & 80 & 2.474 & 544 & 600 & 250 & Dimple-like \\
\hline
\end{tabular}

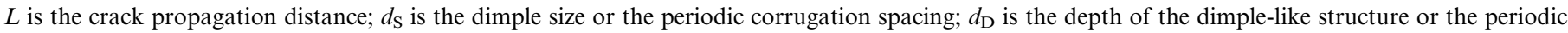

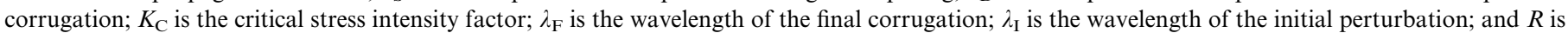
the fracture process zone size.

Taken from ${ }^{\mathrm{a}}$ Fig. 4a, ${ }^{\mathrm{b}}$ Fig. 4c, ${ }^{\mathrm{c}}$ Fig. 4e, ${ }^{\mathrm{d}}$ Fig. 4g, ${ }^{\mathrm{e}}$ Fig. 5a, ${ }^{\mathrm{f}}$ Fig. 5c, ${ }^{\mathrm{g}}$ Fig. 5e, ${ }^{\mathrm{h}}$ Fig. 5g.

pressure can induce instability in the fluid meniscus, i.e. induce an initial perturbation with wavelength $\lambda_{\mathrm{I}}$ in front of the crack.

Once the crack propagation is moved, the negative pressure will pull the unstable fluid meniscus (i.e. the initial perturbation) to advance along the crack propagation direction ( $X$-axis), which can be envisioned as a finger penetrating into the fracture process zone (Fig. $7 b$ ). Once the perturbation front encounters the boundary of the fracture process zone, its development will be stopped in the $X$-axis. However, the extension of the perturbation in the $Y$-axis on the $X-Y$ plane will keep going, and then the dimple-like structure is formed.

If the initial perturbation is frustrated, the fluid meniscus in the crack tip will keep stable. In this case, damage cavities are expected form in the fracture process zone. These damage cavities expand along the $Y$-axis on the $X_{-}$ $Y$ plane, and then coalesce to form a periodic corrugation pattern (Fig. 7c).

\subsection{Fluid meniscus instability criticality}

In this section, the fluid meniscus instability criticality is used to elucidate quantitatively the formation mechanism of fracture surface marks, as shown in Fig. 7.

The primary condition for the crack propagation in the Mg-based BMG is the formation of the fluid meniscus. As mentioned in Section 4.1, local temperature elevation accompanying plastic deformation is the main reason for softening of the glassy phase [35-38]. In the present study, as the crack runs, the maximum temperature rise $u$ could be estimated by a function [39].

$u=\left\lfloor 1.414\left(1-v^{2}\right) K \sigma_{Y} \sqrt{V}\right\rfloor /(E \sqrt{\rho c k})$ where $K$ is the local stress intensity factor $\left(\sim 2 \mathrm{MPa} \mathrm{m}^{0.5}\right), \rho$ is the mass density $\left(3.79 \times 10^{6} \mathrm{~g} \mathrm{~m}^{-3}\right), c$ is the thermal capacity, $\sigma_{\mathrm{Y}}$ is the yield stress $(736 \mathrm{MPa}), E$ is the elastic modulus $(44.7 \mathrm{GPa}), v$ is the Poisson ratio $(0.313)$, and $k$ is the thermal conductivity. For BMG, the thermal capacity roughly equals $1 \mathrm{~J}\left(\mathrm{~g} \mathrm{~K}^{-1}\right.$ [40], and the thermal conductivity is $\sim 5 \mathrm{~W}(\mathrm{~m} \mathrm{~K})^{-1}$, which is weakly composition dependent [41,42]. The maximum crack speed $V$ should not exceed the Rayleigh wave speed $\left(V_{\mathrm{R}}\right)\left(V_{\mathrm{R}} \approx 0.9225 c_{\mathrm{s}}\right.$, where $c_{\mathrm{s}}$ is the shear wave speed $\left.\left(2254 \mathrm{~m} \mathrm{~s}^{-1}\right)\right)$ [43]. The maximum temperature rise is $\sim 438 \mathrm{~K}$, which is slightly higher than the melting temperature $(717 \mathrm{~K})$. In real metallic materials, the crack propagation speed just about approaches $0.73 V_{R}$. As such, the local temperature elevation actually is less than the melting temperature, while it is larger than the glassy transition temperature $(424 \mathrm{~K})$. So it is obvious that the local temperature elevation results in the formation of the viscous fluid matter in the fracture process zone.

Once the fluid matter is formed in the crack front, due to the balance between the negative pressure $\left(\sigma_{\mathrm{N}}\right)$ and the surface energy $(\chi)$, the meniscus of the fluid between two rigid opposite fracture surfaces is assumed to reflect the advance of the crack tip along the $X$-axis (Fig. 7a) [44]. Driven by the negative pressure, the fluid meniscus is in an unstable state. An infinitesimal initial unstable perturbation with a wavelength of $\lambda$ on the meniscus is activated and penetrates into the crack front on the $X-Y$ plane, i.e. the fracture surface plane [45,46], as shown in Fig. 7a. If the wavelength of the initial perturbation $\left(\lambda_{\mathrm{I}}\right)$ is larger than a critical value, i.e.

$\lambda_{I} \geq 2 \pi \sqrt{\frac{\chi}{d \sigma_{N} / d x}}$ 
a

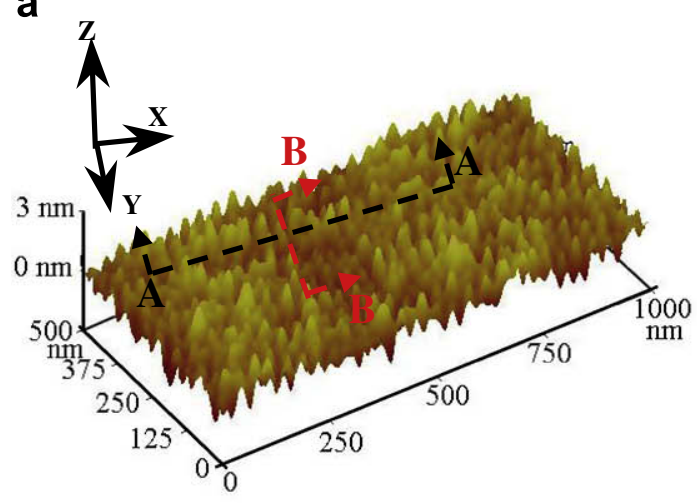

b
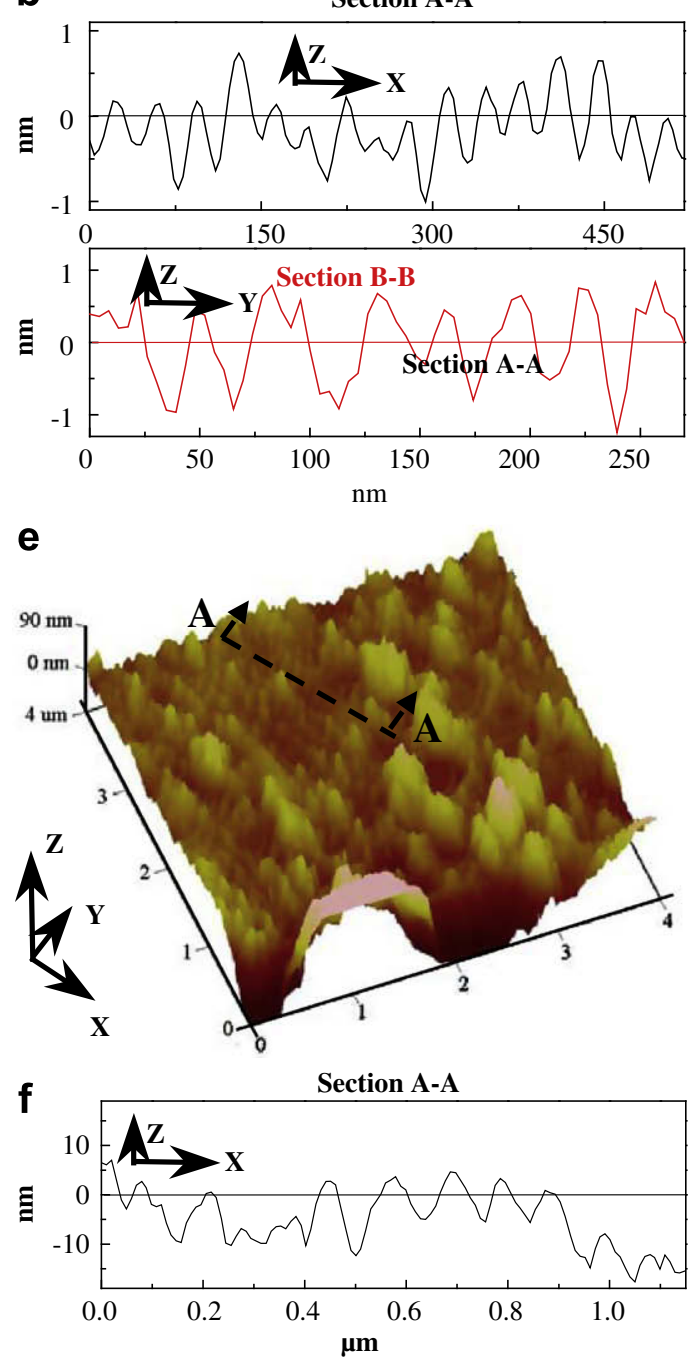

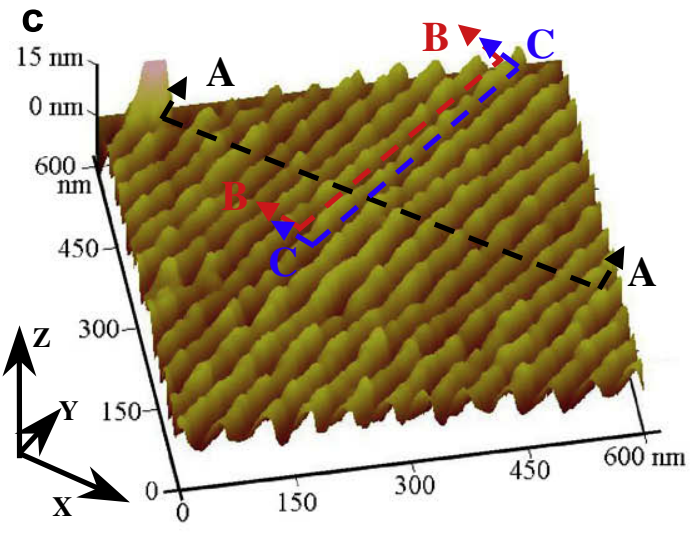

d
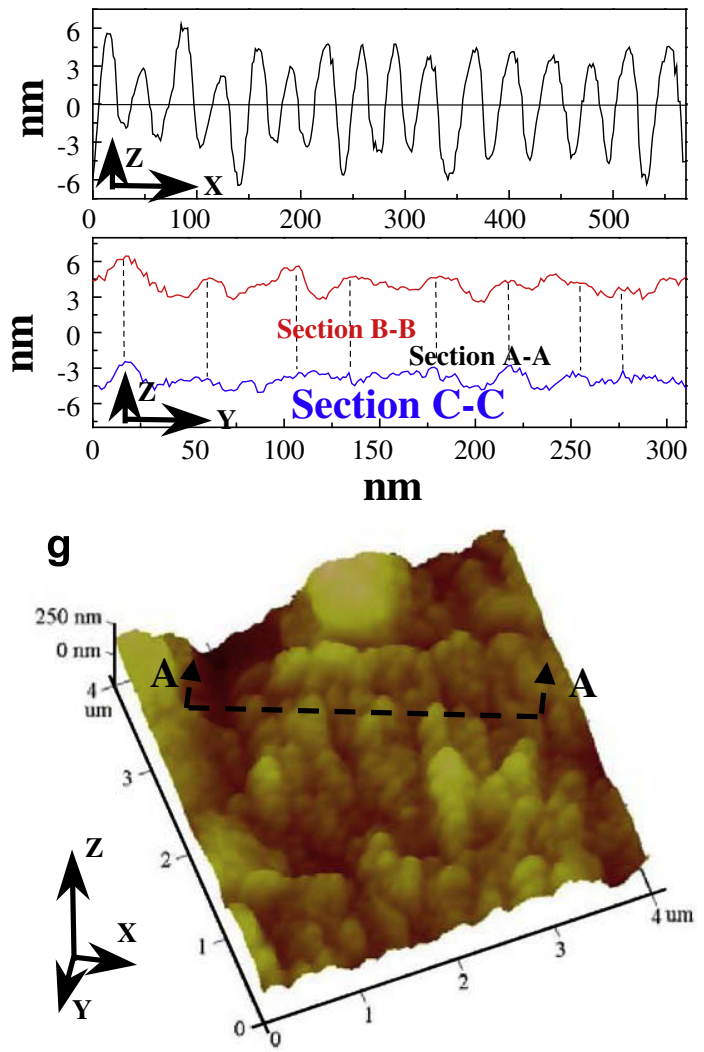

h

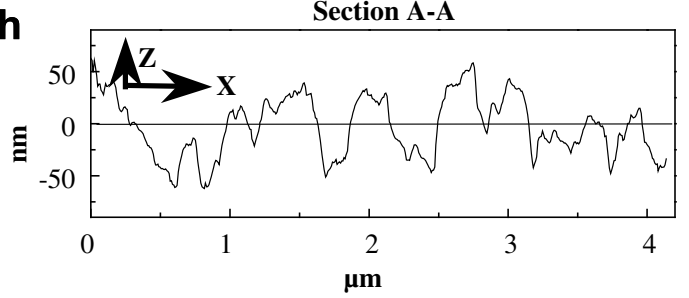

Fig. 6. AFM images for the fractography of the tension fractured BMG. Crack propagation direction is along the $X$-axis. (a) 3D morphology for the corrugation pattern at the crack nucleation spot. (b) Section A-A is sectional shape of the corrugation shape in (a). Section B-B is the sectional shape of the peak for the corrugation in (a). (c) 3D morphology for the corrugation zone. (d) Section A-A is sectional shape of the corrugation in (c). Section B-B and Section $\mathrm{C}-\mathrm{C}$ are the sectional shapes of the peak and the valley for the corrugation in (c), respectively. (e) 3D morphology for the mixture zone. (f) Section A-A is the sectional shape of the dimple-like structure in (e). (g) 3D morphology for the dimple-like structure. (h) Section A-A is the sectional shape of the dimple-like structure in (g).

the initial perturbation will grow [34]. The fluid meniscus exists in the sharp crack tip, which can be assumed to be a fluid flowing in a channel with height $H$. By analyzing the velocity profile on the meniscus, the dominant wavelength of the perturbation $(\lambda)$ can be deduced as [45] 


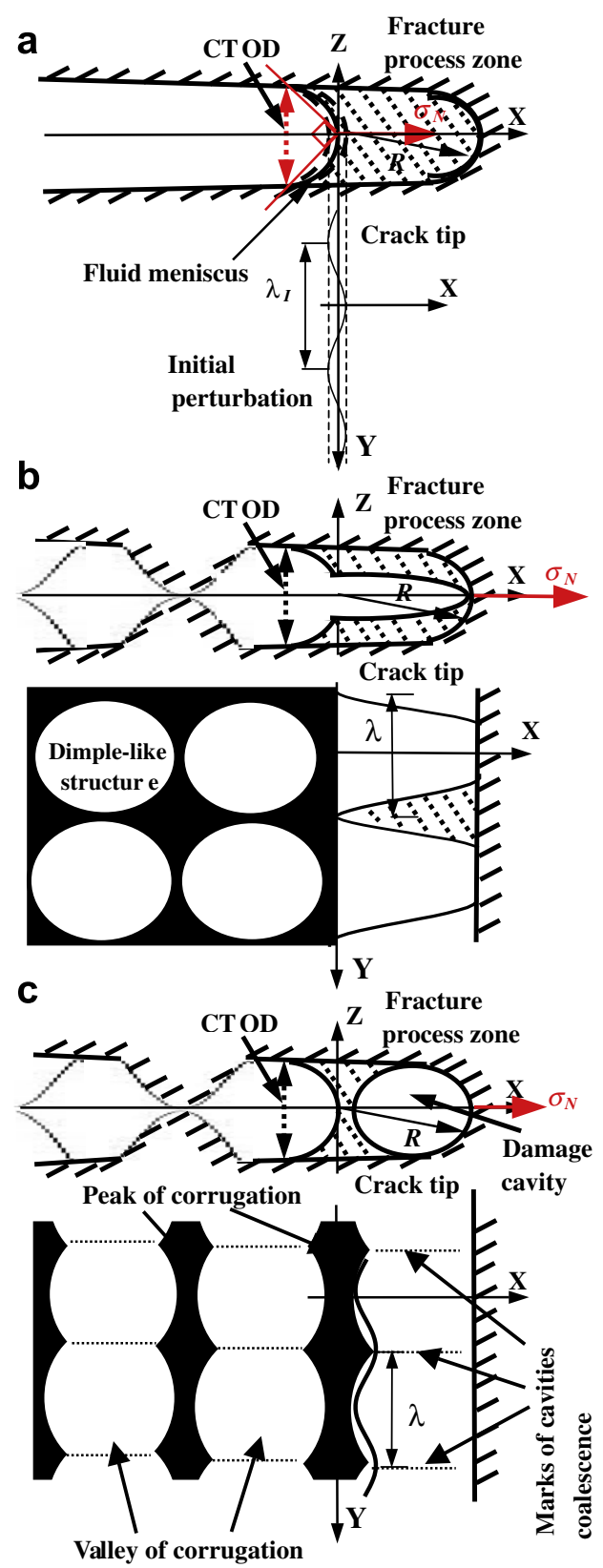

Fig. 7. Sketch of the crack propagation process. Crack propagation direction is $X$-axis and the $X-Y$ plane is the fracture surface plane. (a) Initial stage of the crack propagation. (b) Formation mechanism of the dimple-like structure. (c) Formation mechanism of the periodic corrugation pattern.

$\lambda=2 \pi \sqrt{\sqrt{3}\left(\frac{H \chi}{\eta_{0}}\right)\left[\frac{n}{2(1+2 n)}\right]^{n}\left(\frac{\sqrt{3} H}{U_{0}}\right)^{n}}$

where $n$ is the non-linear exponent of fluid, $U_{0}$ is the average integrated flow velocity of the whole meniscus profile, and $\eta_{0}$ is the viscosity coefficient. Once the initial perturbation is developed, the perturbation on the unstable meniscus will be shaped as a steady state furrow with a steady wavelength of $\lambda_{\mathrm{S}}$, which can be expressed as [45]

$\lambda_{S}=12 \pi^{2} A(n) \frac{\chi}{\tau_{Y}}$ where $\tau_{\mathrm{Y}}$ is the yield shear stress, and $A(n)$ is the wavelength coefficient, which depends on the non-linearity exponent $(n)$ [45]. If this assumption is applied in the present study, the height of the channel roughly equates to the CTOD, which can be expressed as

$H=\mathrm{CTOD}=24 \pi^{2} B(n)\left(\frac{\chi}{\tau_{Y}}\right)$

where $B(n)$ is the CTOD coefficient which is associated with the non-linear exponent $(n)$. In the small-scale yielding mode, the CTOD depends on the critical stress intensity factor $\left(K_{\mathrm{C}}\right)$, i.e.

$\frac{K_{C}^{2}}{E}=m \sigma_{Y} \mathrm{CTOD}$

where $m$ is a dimensionless constant that depends on the material properties and the stress states and $\sigma_{\mathrm{Y}}$ is the yield stress. According to the instability of the fluid meniscus, the relationship of the critical stress intensity factor $\left(K_{\mathrm{C}}\right)$ and the CTOD can be established as [45]

$K_{C}=\sqrt{\mathrm{CTOD} m \sigma_{Y} E}=\sqrt{\frac{24 \pi^{2} B(n) \sqrt{3} \chi E}{2.7}}$

According to the Taylor's meniscus instability criterion, once the wavelength of the developing perturbation $(\lambda)$ is larger than a critical value, i.e. $\lambda_{S} / \sqrt{3}$, the perturbation will develop to form a steady furrow [45,47]. Referring to Fig. 7b, it is obvious that the development of the perturbation can be envisioned as a finger advancing into the fracture process zone, which results in a dimple-like structure being formed. However, if the $\lambda$ value is smaller than the $\lambda_{S} / \sqrt{3}$ value, the development of the perturbation should stop, and the coalescence of the damage cavities will result in the periodic corrugation pattern being formed (cf. Fig. 7c).

Furthermore, the development of the initial perturbation in the meniscus instability cannot pass over the boundary of the fracture process zone. The formation of the damage cavities and coalescence are also confined to the fracture process zone (cf. Fig. $7 \mathrm{~b}$ and c). So the size of the fracture process zone determines the size of the dimple-like structure and the spacing of the periodic corrugation pattern. The small-scale yielding model indicates that the fracture surface separation process is confined to a frontal fracture process zone [32]. The radius of the fracture process zone $(R)$ for plane strain can be estimated as

$R=\frac{1}{6 \pi}\left(\frac{K_{C}}{\sigma_{Y}}\right)^{2}$

Based on the Irwin crack tip stress field solution and the fracture process zone size calculation, i.e. Eq. (8), the negative pressure in the fracture process zone is equivalent to the stress component along the $X$-axis $\left(\sigma_{\mathrm{xx}}\right)$, which can be expressed as [32]

$\sigma_{x x}=\frac{K_{C}}{\sqrt{6 \pi x}} \cos (\theta / 2)[1-\sin (\theta / 2) \sin (3 \theta / 2)]$ 
where $x$ is the distance to the crack tip, and $\theta$ is the degree between the stress component and the crack propagation direction. As the negative pressure is along the $X$-axis, the $\theta$ value is zero, and then $\sigma_{N}=\sigma_{x x}=K_{C} / \sqrt{6 \pi x}$. The derivative of the $\sigma_{\mathrm{xx}}$ is the negative pressure gradient of $\mathrm{d} \sigma_{\mathrm{N}} / \mathrm{d} x$.

\subsection{Application of the fluid meniscus instability to the fracture behavior of the Mg-based BMG}

\subsubsection{Determination of the parameters for the fluid meniscus instability}

The change in local $K_{\mathrm{C}}$ value, as the crack propagates, is evaluated to reflect the dynamic behavior of in the crack propagation. In Eq. (7), the $E$ value is measured from Fig. 1a $(44.7 \mathrm{GPa})$; the $\sigma_{\mathrm{Y}}$ value is equivalent to the $\sigma_{\mathrm{S}}$ value (736 MPa), because the yield behavior and the fracture behavior happen almost simultaneously in the brittle BMG [48]; the $m$ value is determined to be 1.16 for BMG [45], and the CTOD value is roughly equal to twice the depth of the surface marks, as shown in Fig. 7a [26]. The depth of the surface marks $\left(d_{\mathrm{D}}\right)$, i.e. the depths of the dimple-like structure and the periodic corrugation pattern, which are measured from the sectional shape I in Figs. 5 and 6 , and the calculated $K_{\mathrm{C}}$ values in different fracture morphology zones for the tension fractured BMG and pre-notched bending fracture BMG, are listed in Table 1 . The calculated $K_{\mathrm{C}}$ values range from $2.6 \mathrm{MPa} \mathrm{m}^{0.5}$ to $0.5 \mathrm{MPa} \mathrm{m}^{0.5}$ (Table 1), which is in accordance with the measurement of the $\mathrm{Mg}$-based $\mathrm{BMG}\left(\sim 2 \mathrm{MPa} \mathrm{m}^{0.5}\right)$. This further confirms that the fluid meniscus instability takes effect during the tension fracture and the pre-notched bending fracture processes. The stress intensity factors as functions of the crack propagation distance for the tension fractured and pre-notched bending fracture BMG are plotted in Fig. 8. Based on the calculated $K_{\mathrm{C}}$ value, the size of the fracture process zone can be calculated through Eq. (8). The calculated $R$ values are listed in Table 1. In the fracture process zone, the negative stress gradient distributions at different crack propagation distances are plotted in Fig. 9 according to Eq. (9). It is obvious that the $\left|\mathrm{d} \sigma_{N} / \mathrm{d} x\right|$ value in the fracture process zone dramatically decreases with increasing distance from the crack tip, and then reaches a stable state. The $\left|\mathrm{d} \sigma_{N} / \mathrm{d} x\right|$ values at the stable state corresponding to different $K_{\mathrm{C}}$ values are listed in the Fig. 9, which shows a decrease with increasing $K_{\mathrm{C}}$ value, i.e. a larger fracture process zone has a smaller negative stress gradient. Substituting $\left|\mathrm{d} \sigma_{N} / \mathrm{d} x\right|$ values to Eq. (2), the wavelengths of the initial perturbation $\left(\lambda_{\mathrm{I}}\right)$ are obtained and shown in Fig. 8 and Table 1. Here, the surface energy $(\chi)$ is $\sim 1 \mathrm{~J} \mathrm{~m}^{-2}$ for the Mg-based BMG [25]. In Eq. (4), the yield shear stress can be roughly estimated by $\sigma_{Y} / \sqrt{3}$, i.e. $\sim 423 \mathrm{MPa}$. Since the BMG exhibits an ideal plastic manner, the $n$ value as an index reflecting the flow behavior is assumed to be zero, and the $A(n)$ value is $\sim 1.3$ [30]. The critical value of $\lambda_{S} / \sqrt{3}$ is calculated to be $\sim 210 \mathrm{~nm}$. In the dimple-like structure zone, the development of the ini- tial perturbation is stopped at the boundary of the fracture process zone. So, the dimple size is treated roughly as the wavelength of the final corrugation $\left(\lambda_{\mathrm{F}}\right)$, referring to Fig. $7 b$. In the corrugation pattern zone, since the development of the initial perturbation is frustrated, the wave shape of the initial perturbation should be the same as that of the final corrugation, which is in the $X-Y$ plane along the peak of the periodic corrugation. Because the wave shape on the $Y-Z$ plane is the same as that on the $X-Y$ plane (cf. Figs. 5e and 6c), the wavelength of the initial perturbation on the $X-Y$ plane (cf. Fig. 7a) is roughly equal to the wavelength on the $Y-Z$ plane (see the sectional shape II in Figs. $5 f$ and $6 \mathrm{~d}$ ). The $\lambda_{\mathrm{F}}$ values, measured from the AFM results in the pre-notched bending case and the tension case, vs the crack propagation distances are plotted in Fig. 8.

\subsubsection{Formation of the dimple-like structure and the periodic corrugation pattern}

From Fig. 8, it is obvious that the $\lambda_{\mathrm{I}}$ values in the dimple-like structure zone are larger than the $\lambda_{S} / \sqrt{3}$ value in both the pre-notched bending fracture and the tension fracture cases. As such, the initial perturbation in the meniscus is unstable and will develop further to prevent the damage cavities from growing along the $Y$-axis on the $X-Y$ plane. The fact that the $\lambda_{\mathrm{F}}$ values in the dimple-like structure zone are smaller than the $R$ values confirms that the initial perturbation development is confined to the fracture process zone, as shown in Fig. $7 \mathrm{~b}$.

In the mixture zone, the wavelength eventually decreases until it becomes less than the $\lambda_{S} / \sqrt{3}$ value in the prenotched bending case, and increases until it becomes larger than the $\lambda_{S} / \sqrt{3}$ value in the tension case. These movements are due to the fact that the stress intensity factor (also the fracture process zone) decreases to prohibit the development of the initial perturbation.

As the crack propagates in the periodic corrugation

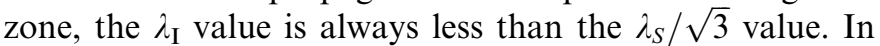
this case, the perturbation is frustrated, and the meniscus in the crack tip keeps stable. The formation of the damage cavities and coalescence will dominate the local fracture behavior. The coalescence of the cavities must leave some marks on the valley of the corrugation pattern, as sketched in Fig. 7c. The sectional shape along the valley of the corrugation pattern is almost the same as that along the peak (see sectional shape II in Figs. 5f and 6d), providing clear evidence of the coalescence of the damage cavities in the fracture process zone. So the corrugation spacing $\left(d_{\mathrm{S}}\right)$ is almost equal to the size of the fracture process zone (Table $1)$.

\subsection{Formation of the pure mirror zone}

As the viscous fluid matter is present in the crack front, the fracture process zone will behave like a viscoelastic body driven by the fast running crack front, and the viscoelastic matter's relaxation time will decrease as the crack 

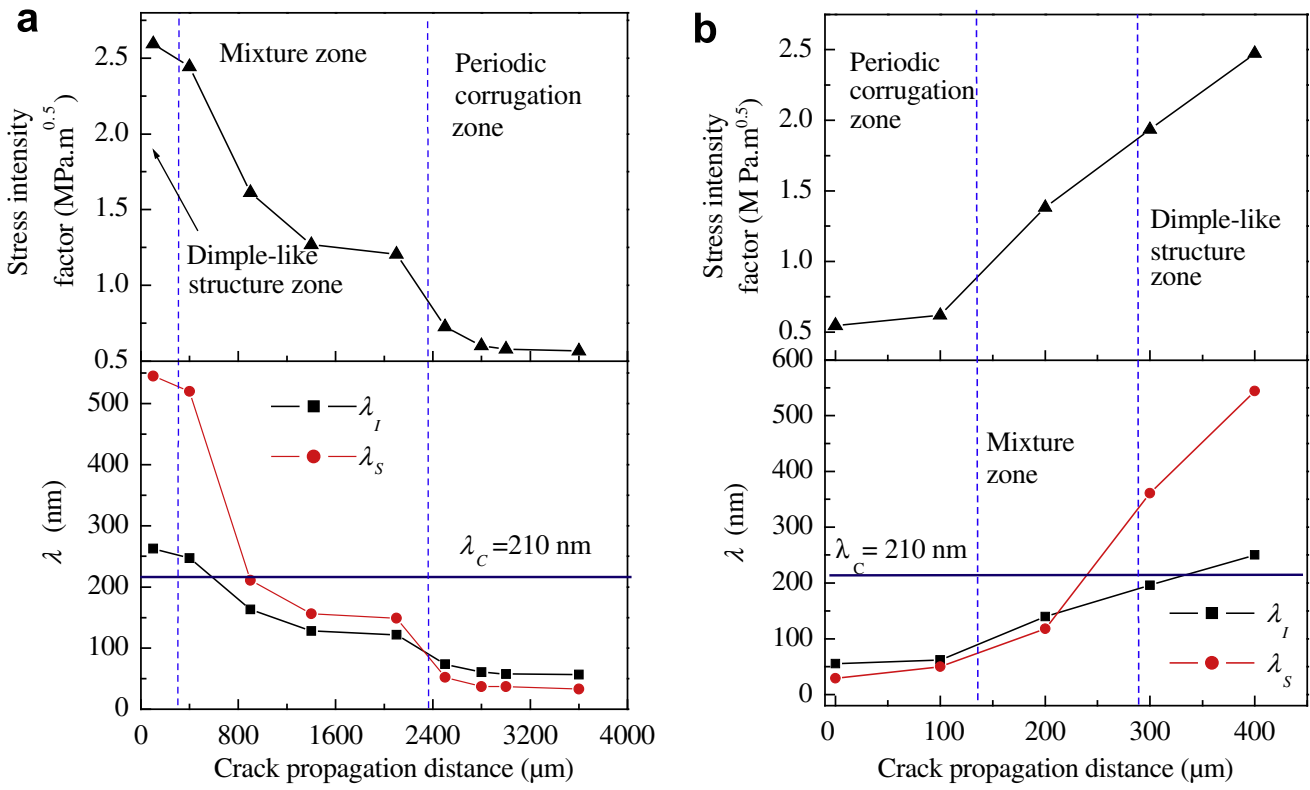

Fig. 8. Wavelength of the initial perturbation $\left(\lambda_{\mathrm{I}}\right)$, wavelength of the final corrugation and stress intensity factor $\left(K_{\mathrm{C}}\right)$ as functions of crack propagation distance.
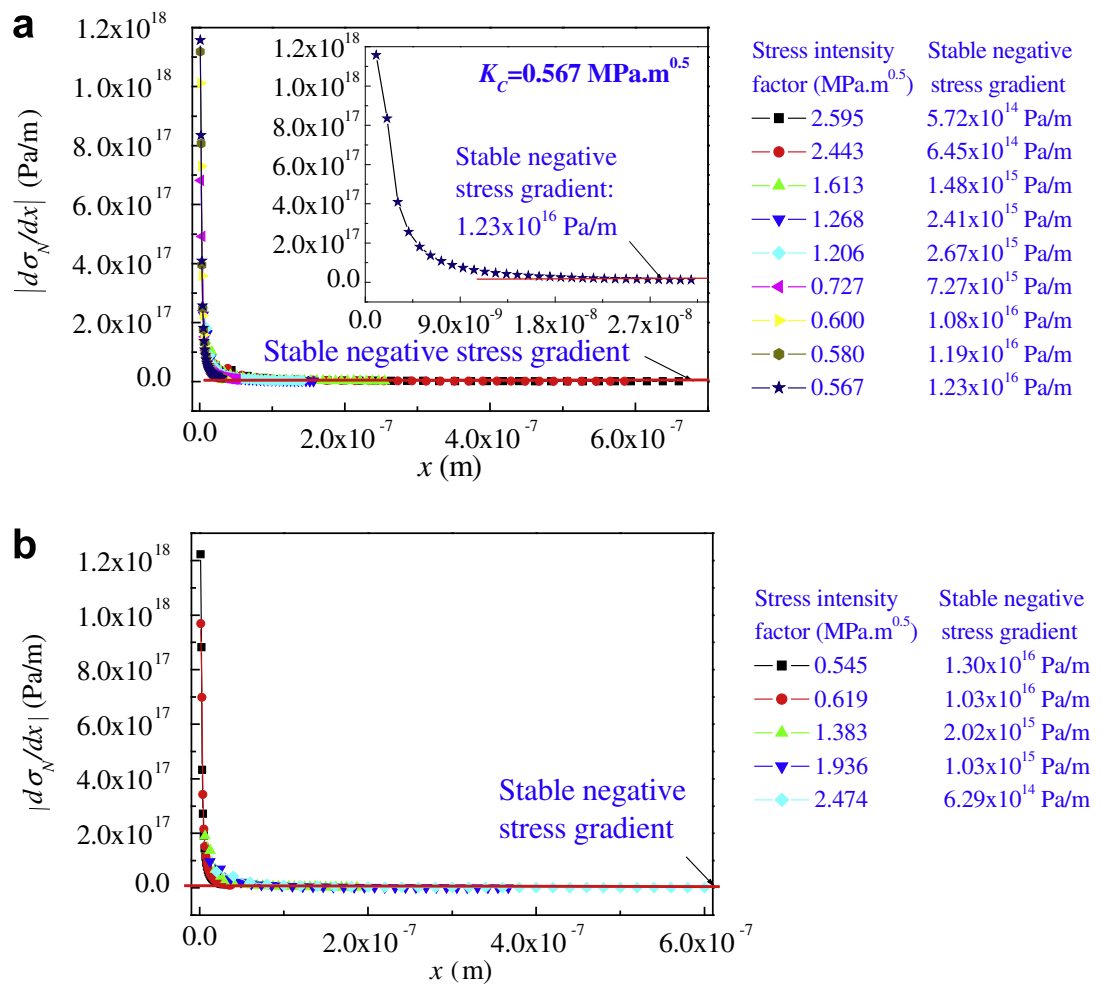

Fig. 9. Negative stress gradient distribution in the fracture process zone at different crack propagation distances. The stable negative stress gradient values are listed on the right of figures. (a) Pre-notched bending fractured BMG and (b) tension fractured BMG. The determination of the stable negative stress gradient is shown in the inset of (a).

propagates $[49,50]$ because, within a continuum of viscoelastic formulation for a propagating crack, a square root singularity is retained, provided that the material is sufficiently crack speed rate sensitive [51]. As the size of the fracture process zone or the periodic corrugation spacing decreases to $\sim 31 \mathrm{~nm}$, the pure mirror zone appears, which means that quasi-cleavage fracture behavior occurs, and the local plastic flow (softening) mechanism is frustrated [30]. In the nanoscale, the general assumption is that the individual atom motion gives way to the cooperative motion of many atoms [52]. In the plastic flow (softening) mechanism, the shear transformation zone (STZ) is a local 
cluster of atoms that undergoes plastic softening to accommodate the shear strain $[53,54]$. The region of one STZ is typically conceived to be about five atoms [53]. For the quasi-cleavage fracture, a tension transformation zone (TTZ) is proposed to describe the crack front with nanoscopically sharp advancing movements by atomic decohesion [30]. The size of the TTZ is assumed to be of the same order as that of the STZ. During the quasi-cleavage fracture process, a local quasi-cleavage zone is believed to be formed in the crack front [41]. Its characteristic length $(L)$ could be expressed as [30]

$L=b\left(\frac{\sigma_{\mathrm{th}}}{\sigma_{Y}}\right)^{2}$

where $b$ is the TTZ spacing, and $\sigma_{\text {th }}$ is the theoretical strength. (As $\sigma_{t h} \cong E / 10$, the $\sigma_{\text {th }}$ is $\sim 4.47 \mathrm{GPa}$.) As the STZ has a dimensional size of $\sim 1 \mathrm{~nm}$ (5 atoms), the TTZ spacing is also $\sim 1 \mathrm{~nm}$. The calculated characteristic length of the quasi-cleavage zone is $\sim 37 \mathrm{~nm}$. This length is very close to the minimum corrugation spacing of $\sim 30 \mathrm{~nm}$ that can be recognized by the AFM and SEM (cf. Figs. 3 and 4). In the bending case, the $R$ value decreases with the crack propagation in the corrugation zone, resulting in an increase in the local tension stress due to stress singularity [7]. It is assumed that the characteristic length of the quasi-cleavage zone is the critical length. Once the $R$ value is reduced to this characteristic length, the local tension stress will approach the theoretical strength and, hence, the quasi-cleavage fracture takes place. In this mirror zone, the energy dissipation mechanism is dominated mainly by the TTZ operation.

\subsection{Influence of the hyperelastic zone on the fractographic evolution}

In the pre-notched bending case, due to the stress concentration in the notch root, a low applied stress can generate the fracture process zone in the notch region, and then the plastic deformation (softening) mechanism dominates the crack propagation. However, in the tension case, the absence of the pre-notch improves the fracture stress. The SEM image has shown that the spacing of the corrugation pattern in the crack origination region is $\sim 35 \mathrm{~nm}$ (Figs. $3 \mathrm{~b}$ and $4 \mathrm{~b}$ ) for the BMG without the notch, which is very close to the quasi-cleavage zone characterization length of $37 \mathrm{~nm}$. It can be reasonably deduced that the crack initiation of the BMG without the notch is dominated by the quasi-cleavage fracture. This quasi-cleavage fracture initiates a penny-like crack in the fracture plane. The stress concentration then occurs in the tip of the penny-like crack. In this case, a viscous fluid meniscus will be formed, as discussed in Section 4.2. Succedent fractographic evolution exhibits the mirror zone, the mist zone and the hackle zone. This fractographic evolution is totally different from that observed in the pre-notched bending case. In general, the transition of the mirror-mist-hackle zones is attributed to the dynamic crack propagation. The crack speed increase will result in dramatic increase in fracture energy, which generates an increase in the fracture surface to consume the surplus of dynamic fracture energy, and then the hackle zone is formed [11], whereas the pre-notched bending fracture suggests that the transition of the mirror-mist-hackle zones does not occur. The bending fracture without notches excludes the loading modes affecting the fractographic evolution. Therefore, it leads to a consideration of the influence of the applied stress.

\subsubsection{Hyperelasticity model}

Generally, the deformation of metals exhibits a perfect linear elasticity in the macroscale, such as the deformation of the Mg-based BMG (cf., Fig. 1a). However, non-linear behavior will occur when the material is failing at the tip of the dynamic crack due to the extreme deformation [55]. A large-scale atomistic simulation provides a hyperelasticity model to depict this non-linear behavior in the dynamic crack tip [17]. This hyperelasticity includes two types of non-linear elastic behavior (Fig. 10a). One is the elastic modulus increasing with strain, called elastic stiffening. The other is the elastic modulus, which decreases with strain, termed elastic softening. The onset of the instability of the dynamic crack propagation is controlled by a competition between the stiffening behavior and the softening behavior [18]. The crack propagation is dominated by the balance between the energy release rate $(G)$ and the newly generated fracture surface energy $(\chi)$, i.e.

$G=\frac{\sigma^{2} r_{H}}{E} f\left(V, c_{1}, c_{2}\right)=2 \chi$

where $\sigma$ is the applied stress, $r_{\mathrm{H}}$ is the radius of the hyperelastic region, and $f\left(V, c_{1}, c_{2}\right)$ is a non-dimensional function of crack speed and wave speeds. Using Eq. (11), it can be found that the crack speed is a function of the ratio of $r_{\mathrm{H}} / \gamma$. The $\gamma$ value is a characteristic length for local energy flux, which is expressed as [17]

$\gamma=\beta \frac{\chi E}{\sigma^{2}}$

where $\beta$ is a coefficient depending on the ratio between hyperelastic and linear elastic properties. The characteristic length for the local energy flux reflects a small region around the crack tip. From this region, energy flows to the moving crack. This concept is shown in Fig. 10b. Through the molecular dynamic simulation, Buehler and Gao et al. $[17,18]$ argued that the competition between the stiffening behavior and softening behavior can be expressed by the ratio of $r_{\mathrm{H}} / \gamma$. If the $r_{\mathrm{H}}$ value is comparable with the $\gamma$ value or the ratio of $r_{\mathrm{H}} / \gamma$ approaches unity, elastic softening dominates crack instability. In this case, the dynamics of the crack is dominated by local elastic properties, i.e. by the hyperelastic softening, because the energy transport required for crack motion occurs within the hyperelastic region. In contrast, if the $r_{\mathrm{H}}$ value is 

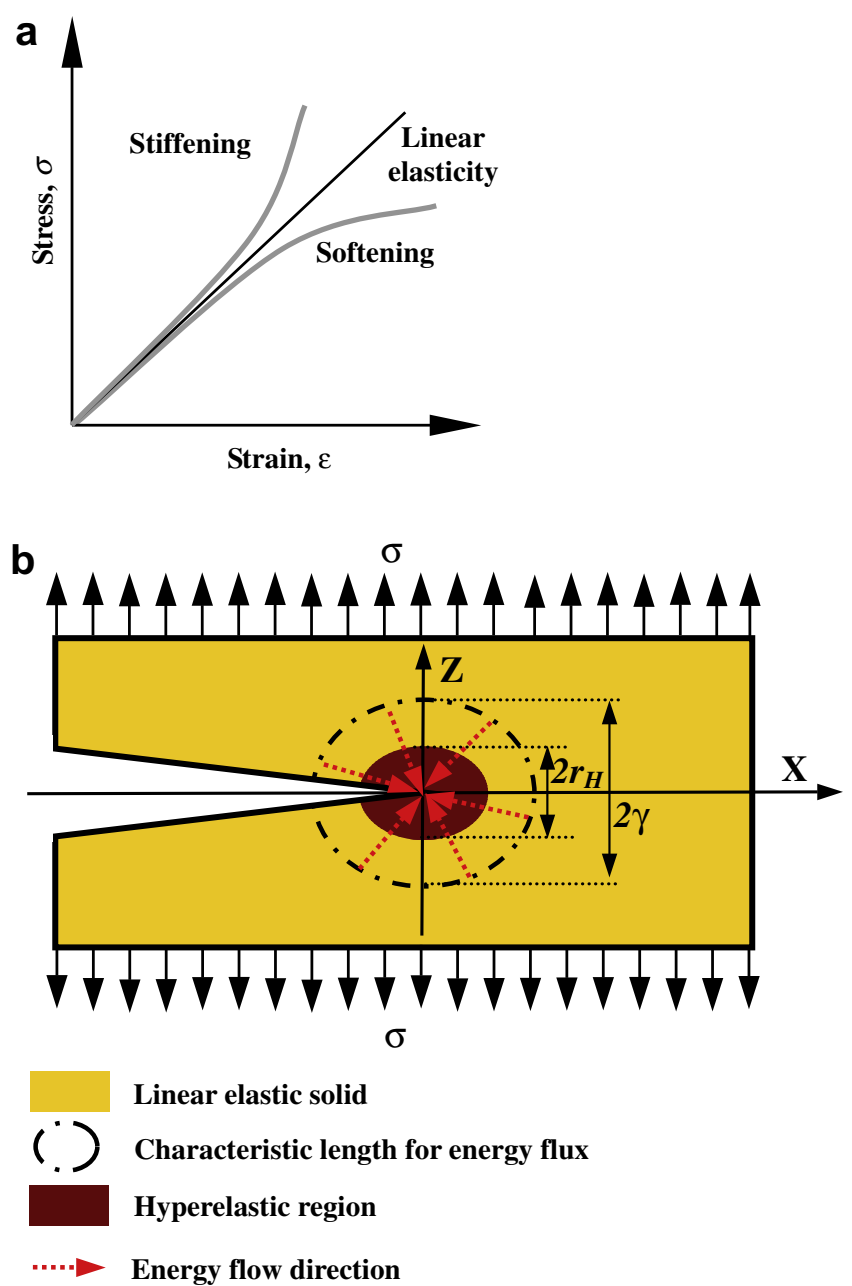

Fig. 10. Sketches illustrate the hyperelastic model: (a) stress-strain relationship of the linear elasticity, elastic stiffening and elastic softening; (b) sketch for the concepts of the hyperelastic region and the characteristic length for energy flux.

smaller than the $\gamma$ value, i.e. $r_{\mathrm{H}} / \gamma \approx 0$, elastically stiffening governs crack instability, in which the crack dynamics is dominated by the global linear elastic properties, as a smaller hyperelastic region cannot afford the energy necessary to guarantee that the dynamic crack propagates. As a consequence, more energy flows into the crack tip from the larger linear elastic region to sustain crack propagation compared with that in elastic softening, i.e. the hyperelasticity plays a reduced role in the instability dynamics [55].

The increase in the $\gamma$ value suggests the domination mechanism of the crack instability transforms from the softening behavior to the stiffening behavior. This transformation results in an increased critical instability speed $\left(V_{\text {inst }}\right)$ for dynamic crack propagation, which is expressed as [18]

$V_{\text {inst }}=V_{\text {shift }}\left(r_{H} / \gamma\right)+\sqrt{\sigma_{\mathrm{coh}} / \rho}$

where $\sigma_{\mathrm{coh}}$ is the cohesion strength, $\rho$ is the density of materials, and $V_{\text {shift }}$ is a shift parameter for the dynamic crack instability speed. This shift is associated with the ratio of
$r_{\mathrm{H}} / \gamma$. As the materials in the pre-notched bending case and the tension case are same, the $\sigma_{\text {coh }}$ value and the $\rho$ value are constant. The $V_{\text {inst }}$ value depends on the $V_{\text {shift }}$ value, which accounts for the relative importance of hyperelastic softening close to the crack tip. If the hyperelastic softening region is large compared with the characteristic length of local energy flux, it will dominate the energy flow, such that the $V_{\text {shift }}$ value will be close to the lower limit, resulting in a small $V_{\text {inst }}$ value. On the contrary, if the softening region is small, the $V_{\text {shift }}$ value will be large, leading to an increased $V_{\text {inst }}$ value.

\subsubsection{Formation of dynamic crack instability}

In the fracture process zone of the Mg-based BMG, the viscous fluid matter exhibits a non-linear stress-strain relationship. Its stress-strain behavior shows a smaller elastic modulus compared with that of a solid around the fracture process zone [56]. So, the fracture process zone is assumed to contain the viscous fluid matter as the hyperelastic region with the size of $r_{\mathrm{H}}$. The characteristic energy length of $\gamma$ is correlated with the surface energy, the elastic modulus and the applied stress. The surface energy can be considered a constant, and the elastic modulus is the linear elastic modulus of $E_{\mathrm{L}}$ (Fig. 10b), i.e. the elastic modulus of the Mg-based BMG, according to the Eq. (12). Then, it is obvious that the $\gamma$ value changes with the square of $\sigma$. In the present study, as the applied stress of $732 \mathrm{MPa}$ in the tension fractured $\mathrm{BMG}$ is six times that of $122 \mathrm{MPa}$ in the pre-notched bending fractured $\mathrm{BMG}$, i.e. the $\gamma$ value in the pre-notched bending case is 36 times larger than that in the tension case. The dynamic behavior of crack propagation in the tension case is dominated by strong softening, and that in the pre-notched bending case is dominated by less strong softening (stiffening). Although it is not able quantitatively to estimate the $\gamma$ value further, one can reasonably assume that, for the pre-notched bending case, much of the energy flows from the linear elastic solids around the fracture process zone into the crack tip to keep the dynamic crack propagating (Fig. 10b). If this is so, the dynamics of the crack motion is dominated by the elastically stiffening. In the tension case, the smaller $\gamma$ value indicates that the energy that flows into the crack tip is less than that in the pre-notched bending case, and the hyperelastic region plays a more important role in the process, dominating the dynamic crack propagation. The energy stored in the hyperelastic region more significantly dominates the crack motion, i.e. the elastic softening (or at least less stiffening) dominates the crack motion. Therefore, the $V_{\text {inst }}$ value in the tension case is reduced and that in the pre-notched bending case is increased. In this case, the crack propagation speed in the tension case can reach the $V_{\text {inst }}$ value more easily compared with that in the bending case. So the crack tip dynamic instability in the tension case generates the hackle zone, and this does not occur in the pre-notched bending case.

Besides the difference of fracture stress resulting in different $V_{\text {inst }}$ values in the pre-notched bending case and in 
the tension case, the change in the $K_{\mathrm{C}}$ value with crack propagation is another factor affecting the $V_{\text {inst }}$ value. According to the Irwin relationship, the fracture energy $(\Gamma)$ in dynamic crack propagation is expressed as [16]

$\Gamma=\frac{1-v^{2}}{E}\left(1-\frac{V}{V_{\mathrm{R}}}\right) K_{I}^{2}$

where $v$ is the Poisson ratio, $V$ is the crack propagation speed, and $V_{\mathrm{R}}$ is the Rayleigh wave speed. In the prenotched bending case, the $K_{\mathrm{C}}$ value decreases, and $V$ increases with crack propagation, suggesting that the $\Gamma$ value decreases with crack propagation. This means that the local energy flux in the crack tip is reduced, and the characteristic energy length must increase [17,57]. In other words, with the $K_{\mathrm{C}}$ value decreasing, the dominating mechanism in crack dynamics is transformation from hyperelastic softening to linear elastic stiffening, i.e. the stiffening behavior is strengthened. The $V_{\text {inst }}$ value is also improved with crack propagating in the bending case. Therefore, the mist zone and hackle zone cannot be formed. In the tension case, the ratio of $r_{\mathrm{H}} / \gamma$ is relative large compared with that in the pre-notched bending case because of the large fracture stress, pointing to a small $V_{\text {inst }}$ value. So the crack tip becomes unstable, and then the hackle zones appear due to the initiation of microbranching. In the hackle zone, the local stress is decreased because the multilayer fracture surface can dissipate the fracture energy flux, and then the energy flux in one layer fracture surface is reduced. In this case, the dominating mechanism of crack dynamics is the same as that which occurs in the bending case.

\section{Conclusions}

The different fractographic evolutions of the $\mathrm{Mg}_{65} \mathrm{Cu}_{25} \mathrm{Gd}_{10}$ BMG, generated by different loading modes, i.e. the pre-notched three-point bending, bending without notch and tension, are observed by the SEM and the AFM. Based on the experimental and analytical results, the following conclusions can be drawn.

(1) The glassy phase in the fracture process zone of the BMG behaves as viscous fluid matter, and the fluid meniscus instability dominates the propagation of the crack. The evolution of the fracture surface is dependent on the wavelength of the initial perturbation and on the size of the fracture process zone.

(2) The dimple structure is developed from the initial perturbation, and a periodic corrugation pattern is formed if the perturbation is frustrated. The quasicleavage fracture occurs when the size of the fracture process zone approaches the characteristic length of the local quasi-cleavage zone.

(3) The fracture energy dissipation mechanisms in the dimple-like structure zone and the corrugation pattern zone are dominated by the local plastic flow (softening) mechanism whereas, in the pure mirror zone, the energy dissipation mechanism is dominated by the quasi-cleavage fracture.

(4) The decreased surface roughness of the brittle BMG during the pre-notched bending test is attributable to the hyperelastic stiffening (or less softening) mechanism which dominates the crack tip instability. The hyperelastic softening mechanism in the tension test results in an increased surface roughness. These findings illustrate that the non-linear behavior in the crack tip plays an important role in controlling the fracture energy dissipation and the crack critical instability speed, which are crucial for understanding the fracture mechanism of brittle BMG.

\section{Acknowledgements}

The work described in this paper was supported by the Research Committee of The Hong Kong Polytechnic University under project code G-YX90 and the NSF of China (Nos. 50621061 and 50731008) and MOST 973 (Grant No. 2007CB613904).

\section{References}

[1] Feinberg J, Gross S, Marder M, Swinney H. Phys Rev Lett 1991;67:457.

[2] Bonamy D, Ravi-Chandar K. Phys Rev Lett 2004;93:099602.

[3] Field JE. Contemp Phys 1971;12:1.

[4] Ramanathan S, Fisher DS. Phys Rev Lett 1997;79:877.

[5] Célarié F, Prades S, Bonamy D, Ferrero L, Bouchaud E, Guillot C, et al. Phys Rev Lett 2003;90:075504.

[6] Guin J-P, Wiederhorn SM. Phys Rev Lett 2004;92:215502.

[7] Freund LB. Dynamic fracture mechanics. Cambridge: Cambridge Univ. Press; 1990. pp. 45, 221.

[8] Sharon E, Fineberg J. Phys Rev B 1996;54:7128.

[9] Livne A, Ben-David O, Fineberg J. arxiv:/arXiv.org:cond-mat/ 06092732006.

[10] Ravi-Chandar K. Int J Fract 1998;90:83.

[11] Sharon E, Fineberg J. Nature (London) 1999;397:333.

[12] Hull D. J Mater Sci 1996;31:1829.

[13] Bouchaud E, Bouchaud JP, Fisher DS, Ramanathan S, Rice JR. J Mech Phys Solids 2002;50:1703.

[14] Sharon E, Cohen E, Fineberg J. Phys Rev Lett 2002;88:085503.

[15] Willis JR, Movchan AB. J Mech Phys Solids 1997;45:591.

[16] Fineberg J. The dynamics of rapidly moving tensile cracks in brittle amorphous material. In: Shukla A, editor. Dynamic fracture mechanics. Hong Kong: World Scientific; 2006. p. 104.

[17] Buehler MJ, Abraham FF, Gao HJ. Nature (London) 2003;426:141.

[18] Buehler MJ, Gao HJ. Nature 2006;439:307.

[19] Xi XK, Wang RJ, Zhao DQ, Pan MX, Wang WH. J Non-Cryst Solids 2004;344:105.

[20] Zheng Q, Cheng S, Strader JH, Ma E, Xu J. Scr Mater 2006;56:161.

[21] Ponnambalam V, Poon SJ, Shiflet GJ. J Mater Res 2004;19:1320.

[22] Chen QJ, Shen J, Zhang DL, Fan HB, Sun JF. J Mater Res 2007;22:358.

[23] Li S, Xi XK, Wei YX, Luo Q, Wang YT, Tang MB, et al. Sci Tech Adv Mater 2005;6:823.

[24] Lewandowski JJ, Wang WH, Greer AL. Philos Mag Lett 2005;85:77.

[25] Xi XK, Zhao DQ, Pan MX, Wang WH, Wu Y, Lewandowski JJ. Phys Rev Lett 2005;94:125510. 
[26] Wang G, Zhao DQ, Bai HY, Pan MX, Xia AL, Han BS, et al. Phys Rev Lett 2007;98:235501.

[27] Zhang ZF, Wu FF, Gao W, Tan J, Wang ZG, Stoica M, et al. Appl Phys Lett 2006;89:251917.

[28] Shen J, Liang WZ, Sun JF. Appl Phys Lett 2006;89:121908.

[29] Xi XK, Zhao DQ, Pan MX, Wang WH, Wu Y, Lewandowski JJ. Appl Phys Lett 2006;89:181911.

[30] Jiang MQ, Ling Z, Meng JX, Dai LH. Philos Mag 2008;88:407.

[31] Schuh CA, Hufnagel TC, Ramamurty U. Acta Mater 2007;55:4067.

[32] Lawn B. Fracture of brittle solids. 2nd ed. Cambridge: Cambridge University Press; 1993. pp. 25, 53-174.

[33] Rice JR, Levy N. In: Argon AS, editor. The physics of strength and plasticity. Cambridge, MA: MIT Press; 1969. p. 286.

[34] Pitts E, Greiller J. J Fluid Mech 1961;11:33.

[35] Spaepen F. Acta Metall 1977;25:407.

[36] Taub AI. Acta Metall 1980;28:633.

[37] Yang B, Morrison ML, Liaw PK, Buchanan RA, Wang GY, Liu CT, et al. Appl Phys Lett 2005;86:141904.

[38] Lewandowski JJ, Greer AL. Nat Mater 2006;5:15.

[39] Flores KM, Dauskardt RH. J Mater Res 1999;14:638.

[40] Newman MEJ. Contemp Phys 2005;46:323.

[41] Li L, Lin T, Dong C, Lin JJ. Phys Rev B 2006;74:172201.
[42] Yamasaki M, Kagao S, Kawamura Y. Scripta Mater 2005;53:63

[43] Ravi-Chandar K, Yang B. J Mech Phys Solids 1997;45:535.

[44] Finnemore EJ, Franzini JB. Fluid mechanics with engineering applications. 10th ed. New York: McGraw-Hill; 2002. pp. 37.

[45] Argon AS, Salama M. Mater Sci Eng 1976;23:219.

[46] Taylor GI. Proc Roy Soc (Lond) 1950;A201:192.

[47] Saffman PG, Taylor GI. Proc Roy Soc (Lond) 1958;A245:312.

[48] Zhang ZF, Eckert J, Schultz L. Acta Mater 2003;51:1167.

[49] Kurtze DA, Hong DC. Phys Rev Lett 1993;71:847.

[50] Wang G, Han YN, Xu XH, Han BS, Ke FJ, Wang WH. J Appl Phys 2008;103:093520.

[51] Suo Z, Shih CF, Varias AG. Acta Metall Mater 1993;41:1551.

[52] Lubarda VA, Blume JA, Needleman A. Acta Metall Mater 1993;41:625.

[53] Argon AS. Acta Metall 1979;27:47.

[54] Falk ML, Langer JS. Phys Rev E 1998;57:71925.

[55] Buehler MJ, Gao HJ. In: Shukla A, editor. Dynamic fracture mechanics. Hong Kong: World Scientific; 2006. p. 8.

[56] Meyers MA, Chawla KK. Mechanical behavior of materials. Upper Saddle River, NJ: Prentice Hall; 1998. pp. 98-103.

[57] Broberg KB. Int J Solids Struct 1995;32:883. 